\title{
Cold Collisions in a Molecular Synchrotron
}

\author{
Aernout P. P. van der Poel, ${ }^{1}$ Peter C. Zieger, ${ }^{1,2}$ Sebastiaan Y. T. van de Meerakker, ${ }^{3}$ \\ Jérôme Loreau, ${ }^{4}$ Ad van der Avoird, ${ }^{3}$ and Hendrick L. Bethlem ${ }^{1}$ \\ ${ }^{1}$ LaserLaB, Department of Physics and Astronomy, Vrije Universiteit, \\ De Boelelaan 1081, 1081 HV Amsterdam, The Netherlands \\ ${ }^{2}$ Fritz-Haber-Institut der Max-Planck-Gesellschaft, Faradayweg 4-6, 14195 Berlin, Germany \\ ${ }^{3}$ Radboud University, Institute for Molecules and Materials, Heijendaalseweg 135, 6525 AJ Nijmegen, The Netherlands \\ ${ }^{4}$ Service de Chimie Quantique et Photophysique, Université Libre de Bruxelles (ULB) CP 160/09, \\ 50 avenue F.D. Roosevelt, 1050 Brussels, Belgium
}

(Received 30 June 2017; published 16 January 2018)

\begin{abstract}
We study collisions between neutral, deuterated ammonia molecules $\left(\mathrm{ND}_{3}\right)$ stored in a $50 \mathrm{~cm}$ diameter synchrotron and argon atoms in copropagating supersonic beams. The advantages of using a synchrotron in collision studies are twofold: (i) By storing ammonia molecules many round-trips, the sensitivity to collisions is greatly enhanced; (ii) the collision partners move in the same direction as the stored molecules, resulting in low collision energies. We tune the collision energy in three different ways: by varying the velocity of the stored ammonia packets, by varying the temperature of the pulsed valve that releases the argon atoms, and by varying the timing between the supersonic argon beam and the stored ammonia packets. These give consistent results. We determine the relative, total, integrated cross section for $\mathrm{ND}_{3}+$ Ar collisions in the energy range of $40-140 \mathrm{~cm}^{-1}$, with a resolution of $5-10 \mathrm{~cm}^{-1}$ and an uncertainty of $7 \%-15 \%$. Our measurements are in good agreement with theoretical scattering calculations.
\end{abstract}

DOI: 10.1103/PhysRevLett.120.033402

The crossed molecular beam technique, pioneered by Herschbach and Lee, has yielded a detailed understanding of how molecules interact and react [1,2]. Until recently, these crossed molecular beam studies were limited by the velocities of the molecular beams to collision energies above a few hundred $\mathrm{cm}^{-1}\left(1 \mathrm{~cm}^{-1} \simeq 1.4 \mathrm{~K}\right)$. Over the past years, however, a number of ingenious methods [3-5] have been developed to study collisions in the cold regime. These advances are important for several reasons. First, the temperatures of interstellar clouds are typically between 10 and $100 \mathrm{~K}$; collision data of simple molecules at low temperatures are thus highly relevant for understanding the chemistry in these clouds [6]. Furthermore, quantum effects become important at low temperatures, where few partial waves contribute and the de Broglie wavelength associated with the relative velocity becomes comparable to or larger than the intermolecular distances. Of particular interest are resonances of the collision cross section as a function of the collision energy [7-10]. The position and shape of these resonances are very sensitive to the exact shape of the PES and thus serve as precise tests of our understanding of intermolecular forces.

The ability to control the velocity of molecules using time-varying electric fields has allowed studies of inelastic collisions of $\mathrm{OH}$ and $\mathrm{NO}$ molecules with rare gas atoms at low collision energies [11-14]. Using cryogenically cooled beams under a small (and variable) crossing angle, inelastic collisions of $\mathrm{O}_{2}$ and $\mathrm{CO}$ with $\mathrm{H}_{2}$ and helium at energies between 5 and $30 \mathrm{~K}$ have been studied [15]. Even lower temperatures can be obtained by using magnetic or electric guides to merge two molecular beams into a single beam. This technique has been used to study Penning ionization reactions of various atoms and molecules with metastable helium [16-19] and collisions between ground state hydrogen molecules and hydrogen molecules in high Rydberg states [20].

In a different approach, trapped ions at millikelvin temperatures are monitored while slow, velocity-selected beams of molecules pass through the trap [21,22]. The ions are stored for a long time and their number can be accurately determined, which allows the study of reactions with rates as small as one per minute. In a similar fashion, collisions of slow beams of ammonia with magnetically trapped $\mathrm{OH}$ molecules were observed [23], as were collisions of slow beams of rare gas atoms and $\mathrm{SF}_{6}$ with lithium in a magneto-optic trap [24].

Here, we study collisions between neutral ammonia molecules stored in a synchrotron and beams of argon atoms. Using a synchrotron for collision studies offers two advantages: First, the collision partners move in the same direction as the stored molecules, resulting in a small relative velocity and thus a low collision energy. Second, the sensitivity to collisions is enhanced by storing the ammonia molecules for many round-trips. Our approach thus combines the low collision energies obtained in experiments that use merged molecular beams [16-20] 


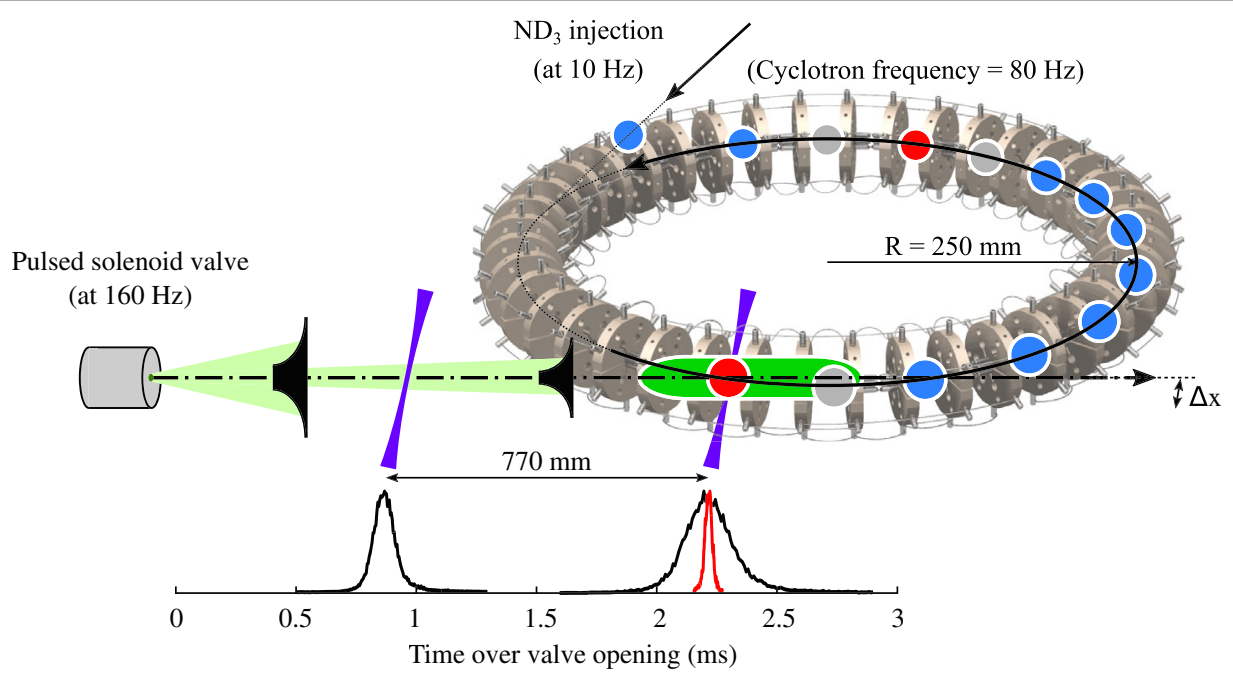

FIG. 1. Schematic view of the synchrotron and beam line. Supersonic beams from a cooled Even-Lavie valve intersect the synchrotron a quarter round-trip downstream from the injection point. The packets of argon (shown in green) are timed such that the probe packets (shown in red) encounter an argon packet on every round-trip, while reference packets (shown in blue) provide a simultaneous measurement of the background loss. Bottom: In order to determine the velocity distribution of the argon beams, the time-of-flight spectra are recorded at two positions along the beam line. For reference, the time-of-flight profile of a stored packet of ammonia is also shown (red curve).

with the high sensitivity of experiments that monitor trap loss [21-24].

The synchrotron used in our experiment is shown schematically in Fig. 1. It consists of 40 electric hexapoles arranged in a circle with a diameter of $0.5 \mathrm{~m}$, to which voltages of up to $\pm 5 \mathrm{kV}$ are applied. With these settings, we store packets of $\mathrm{ND}_{3}$ molecules in the low-field-seeking sublevel of the $J=1, K=1$ rovibrational ground state with velocities in the range of $100-150 \mathrm{~m} / \mathrm{s}$. At any time during the experiment, 14 packets of ammonia molecules are held by the synchrotron. Each packet of this train is stored for up to $1.2 \mathrm{~s}$, while at a $10 \mathrm{~Hz}$ rate new packets are being injected in front of the train and packets at the back of the train are being detected. More details on the synchrotron and injection beam line can be found in Zieger et al. [25-27].

The stored packets of ammonia molecules are made to collide with beams of argon atoms released by a pulsed Even-Lavie valve [28], which can be operated at temperatures between $-150^{\circ}$ and $+30^{\circ} \mathrm{C}$. The pulsed valve is operated at $160 \mathrm{~Hz}, 2$ times the cyclotron frequency of the stored ammonia packets, such that every tenth $\mathrm{ND}_{3}$ packet will encounter a fresh Ar packet every round-trip. We will refer to these packets as probe packets. Packets that do not encounter the argon beam, referred to as reference packets, provide a simultaneous measurement of the background loss. Collisions between stored ammonia molecules and argon atoms will (almost always) cause ammonia molecules to be lost from the trap, as the longitudinal and transverse trap depths are much smaller than the collision energies. The argon beam is displaced from the equilibrium orbit of the stored ammonia molecules by about $1.6 \mathrm{~mm}$ such that it intersects the path of the ammonia molecules twice. This simplifies the analysis (vide infra).

As shown in Fig. 1, the relative intensities of the Ar beams are monitored at two positions: $480 \mathrm{~mm}$ downstream from the valve and inside the detection zone of the synchrotron, $1250 \mathrm{~mm}$ downstream from the valve. The argon atoms are detected by $3+1$ resonantly enhanced multiphoton ionization (REMPI) via the $3 s^{2} 3 p^{5}\left({ }^{2} P_{1 / 2}\right) 4 s$ state [29] using a pulsed UV laser running at $314 \mathrm{~nm}$. The arrival time distributions measured at the two detection zones are used to derive the longitudinal velocity distributions. The black curves in Fig. 1 show typical time-offlight profiles measured in both detection zones for an argon beam at a valve temperature of $-150{ }^{\circ} \mathrm{C}$. For reference, the time-of-flight profile of a packet of ammonia after making 90 round-trips is also shown (red curve).

Fig. 2 shows the number of ammonia molecules in the probe beam (red squares) and reference beam (blue squares) as a function of the storage time. Note that the error bars reflecting the statistical spread of the ion signal are (in most cases) smaller than the symbols. In this particular experiment, hydrogen molecules are used as a collision partner. The solid lines show the result of fits to the data using the expression $n=n_{0} e^{-k \cdot R T}$, with $n$ the number of detected ions per shot, $R T$ the number of roundtrips the packets have made before being detected, and $k$ the loss rate. For the reference packets, we find a loss rate of $1.41 \pm 0.08 \%$ per round-trip, corresponding to a lifetime of $1.0 \mathrm{~s}$. For the probe packets, we find a loss rate of $2.67 \pm$ $0.11 \%$ per round-trip, which implies that collisions with the supersonic beam induce an additional loss rate of $1.26 \pm$ $0.14 \%$ per round-trip. 


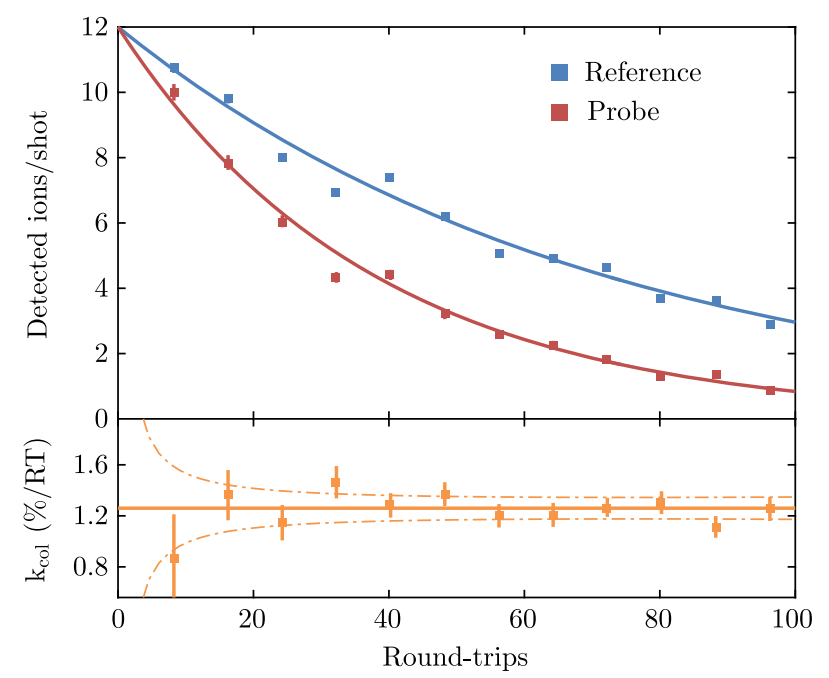

FIG. 2. Upper frame: Number of detected $\mathrm{ND}_{3}$ ions per shot as a function of the number of round-trips spent in the synchrotron, for both the probe (red) and the reference packets (blue). Each data point is an average over 3240 (reference) or 360 (probe) shots. The solid lines denote exponential fits. Lower frame: From ratios of the numbers of detected ions, the loss rate due to collisions can be determined at each number of round-trips. The dash-dotted lines show the expected statistical uncertainty based on the number of detected ions per shot.

The orange data points in the lower panel in Fig. 2 show the loss rate due to collisions calculated from the number of ions detected in the probe and reference beams at specific round-trip numbers, using $k_{\text {col }}=-\ln \left(n_{\text {probe }} / n_{\text {ref }}\right) / R T$. The error bars reflect the statistical spread of the ion signal. The uncertainty of $k_{\mathrm{col}}$ decreases dramatically during the first round-trips and reaches an optimum after $\sim 70$ roundtrips, at which point only $14 \%$ of molecules in the probe beam remain. When the storage time is increased further, the uncertainty of the measured probe beam intensity becomes the limiting factor and the uncertainty of $k_{\mathrm{col}}$ increases. The orange dash-dotted lines show the expected statistical uncertainty, assuming the number of detected ions is governed by Poisson statistics, which are in good agreement with the experimental results. Note that the deviations from a perfect exponential decay of the probe and reference signals observed in the upper panel in Fig. 2 are absent in the extracted loss rate shown in the lower panel. This is a crucial feature of our method: Fluctuations due to, for instance, intensity and/or wavelength drifts of the laser, temperature variations of the valve, or collective oscillations of the packet inside the synchrotron are common to the reference and probe signals and are divided out.

Of critical importance is the delay between the trigger of the valve that releases the argon atoms and the arrival time of the $\mathrm{ND}_{3}$ probe packet in the detection zone. This delay determines whether the ammonia molecules collide with atoms located more in the leading or trailing end of the argon packet or, in fact, whether they collide at all.
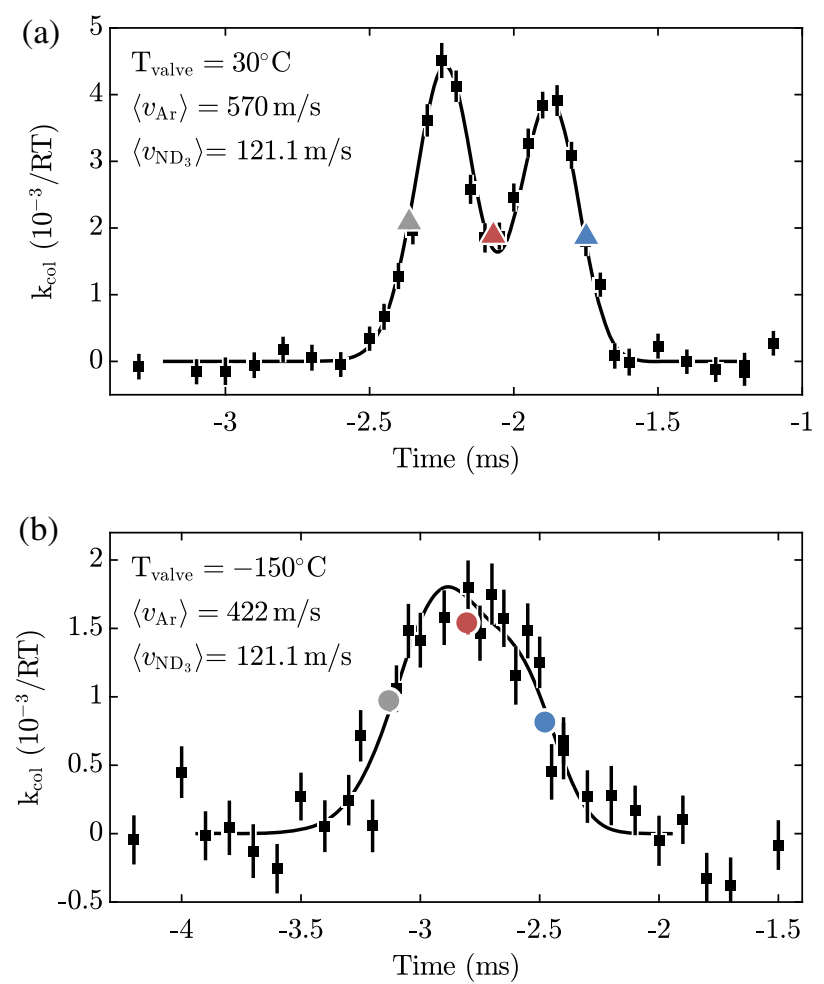

FIG. 3. Loss rate due to collisions with argon atoms versus valve timing for two different valve temperatures. The black squares consist of 2400 shots each and the colored points of 21600 shots each. The lines depict results of a simulation of the experiment, individually scaled to fit the data (see the main text).

Furthermore, as the flight time from the valve to the synchrotron is much larger than the opening time of the valve, there is a strong correlation between the position of the argon atoms and their velocity. Hence, the delay determines the velocity of the argon atoms that are encountered by the ammonia beam.

The loss rates of $\mathrm{ND}_{3}$ molecules (with velocities of 121.1 and $138.8 \mathrm{~m} / \mathrm{s}$ ) due to collisions with argon atoms (with velocities around 570, 524, 475, and $422 \mathrm{~m} / \mathrm{s}$ ) were measured as a function of the aforementioned delay. Each data point is the result of 2400 shots-corresponding to a measurement time of $4 \mathrm{~min}$. To be robust against possible drifts of the Ar beam density, the data were taken while toggling between the two ammonia speeds after every data point and picking the timings from a list in a random order. The results for an ammonia velocity of $121.1 \mathrm{~m} / \mathrm{s}$ colliding with beams of argon with an average velocity of 570 and $422 \mathrm{~m} / \mathrm{s}$ are shown in Fig. 3. The measurements can be seen to feature two peaks, resulting from the fact that the argon beam intersects the synchrotron at two distinct locations. These peaks become less well resolved as the argon packets become slower and concomitantly longer.

Additional data were taken at three specific timings for both ammonia velocities. These data are shown as the colored symbols in Fig. 3. Each of these is the result of 
21600 shots, corresponding to a measurement time of 36 min per point. To detect and correct for possible drifts of the Ar beam density, we cycled nine times through the six different configurations. No significant drifts were detected.

The solid curves in Fig. 3 show results of a simulation of our experiment. The simulation uses as input (i) the longitudinal position and velocity distributions of the argon beams taken from the time-of-flight profiles at two locations, (ii) the shape and size of the cross section of the argon beams taken from measurements where the height of the laser beam was scanned, from measurements where the position of the valve was scanned, and from the geometry of our beam machine, (iii) the horizontal displacement of the argon beam from the equilibrium orbit determined from the time difference between the maxima in the measured loss rates, and (iv) the velocity and equilibrium radius of the synchronous molecule taken from simulations of the synchrotron [27]. For each combination of valve temperature and ammonia velocity, the simulated curves are scaled vertically to fit the black data points in Fig. 3. As seen from the figure, the simulations describe the measurements very well, which confirms that we have an excellent understanding of the experiment.

The scaling factors obtained by fitting the simulations to the measurements are the products of the collision cross sections and the absolute column densities of the argon beams at the corresponding valve temperatures. Hence, by dividing the scaling factors by the relative column densities of the argon beams, derived from time-of-flight measurements in the detection zone of the synchrotron, we obtain the relative total cross sections. We correct for the fact that a small but significant fraction of the elastic collisions does not eject molecules from the ring; see Supplemental Material [30]. The resulting cross sections are shown as the black data points in Fig. 4. The blue, red, and gray points in Fig. 4 are found by scaling the simulations to the

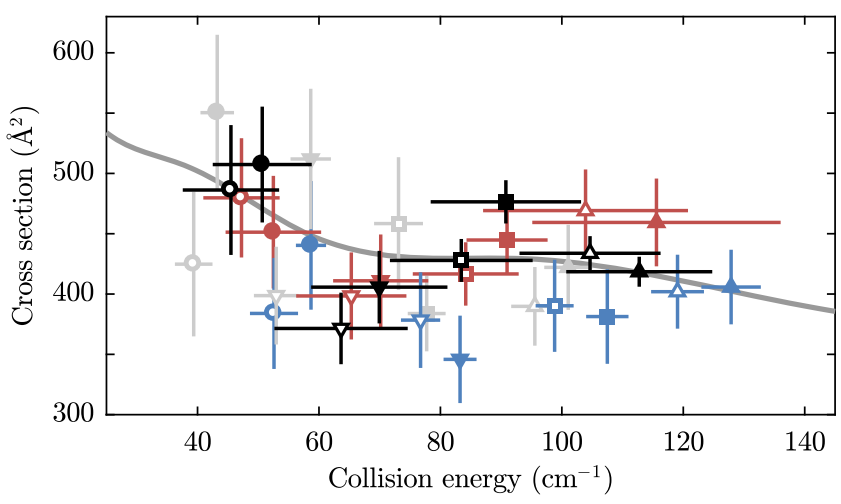

FIG. 4. Total, integrated, $\mathrm{ND}_{3}+\mathrm{Ar}$ collision cross section versus collision energy. The collision energy is varied in three different ways (see the main text). The measurements are collectively fit to a theoretical calculation [31] (solid line) with a single global scaling factor. data points measured at the front, center, and back of the argon packet, respectively (the colored points shown in Fig. 3). The vertical error bars represent the statistical uncertainties of the measurements (the standard deviation), ranging from $7 \%$ to $15 \%$, while the horizontal error bars represent the spread in collision energy, retrieved from the simulations, and range from 5 to $10 \mathrm{~cm}^{-1}$. The data taken in the fast part of the beam (the blue data points) have the best energy resolution. The data points taken by averaging over the entire velocity distribution have smaller uncertainties, but their energy resolution is worse. The collision cross sections determined from the different data sets agree with each other within their combined errors, although the cross sections retrieved from colliding ammonia molecules with the fast part of the argon beam (blue data points) appear to be systematically smaller than the cross sections retrieved from colliding ammonia molecules with the slow or center part of the argon beam.

The solid line depicted in Fig. 4 is the result of theoretical calculations described in Ref. [31], convoluted with a normal distribution with a standard deviation of $5 \mathrm{~cm}^{-1}$. The measurements are collectively fit to this calculation with a single global scaling factor that represents the density of the argon beam at $T=-150^{\circ} \mathrm{C}$, which is found to be $7.8 \times 10^{9} \mathrm{~cm}^{-3}$. This density is in agreement with a crude estimate of the density from the REMPI measurements. In the future, we plan to measure the density more accurately using a femtosecond laser [32]. Although the $\mathrm{ND}_{3}+$ Ar collision cross section in this energy range does not show spectacular features, the shallow minimum around $70 \mathrm{~cm}^{-1}$ predicted by the theory is reproduced in the experiment.

In conclusion, we have performed collision experiments between argon atoms in a supersonic beam and $\mathrm{ND}_{3}$ molecules stored in a synchrotron. Our measurements demonstrate that storing molecules for many round-trips increases the sensitivity dramatically, and that copropagating beams allow low collision energies to be studied, hence providing a robust and general method to measure the total cross section for low energy collisions. Our method has a number of additional features that make it attractive. (i) By comparing packets that are simultaneously stored in the synchrotron, the measurements are independent of the ammonia intensity and immune to variations of the background pressure in the synchrotron. (ii) As the probe packets interact with many argon packets, shot-to-shot fluctuations of the argon beam are averaged out. By toggling rapidly between different ammonia velocities and timings, slow drifts of the argon beam intensity are eliminated.

The collision energy is currently limited by the large difference between the velocity of the stored molecules and the velocities in the supersonic beam. Lower collision energies could be reached by using molecules from cryogenically cooled beams as a collision partner [33] and/or by using a larger synchrotron which would be able 
to store ammonia molecules at a higher velocity. Ideally, a synchrotron would be used that can store molecules directly from a supersonic beam without deceleration. Furthermore, by storing beams both clockwise and anticlockwise in the synchrotron, it is possible to measure collision energies from 0 to $2000 \mathrm{~cm}^{-1}$. Note that, if the velocities of the beams are more similar, the energy resolution will be improved [34], ultimately limited to $\sim 10 \mathrm{mK}$, the temperature of the stored ammonia packets. A higher resolution can also be obtained (at the cost of increasing the collision energy) by crossing the beams at right angles. This would make it possible to resolve the fine structure on the elastic cross section due to scattering resonances [31]. Finally, collision studies with paramagnetic molecules and atoms, such as hydrogen, could be performed in a magnetic synchrotron [35].

We thank Chris Eyles for help with setting up the synchrotron after being moved to Amsterdam. We thank Rob Kortekaas for technical support and Wim Ubachs and Gerard Meijer for helpful discussions and support. This research has been supported by the Netherlands Foundation for Fundamental Research of Matter (FOM) via the program "Broken mirrors and drifting constants." S. Y. T. v. d. M. acknowledges support from the European Research Council under the European Union's Seventh Framework Program (FP7/2007-2013)/ERC Grant Agreement No. 335646 MOLBIL.

[1] Y. T. Lee, Science 236, 793 (1987).

[2] D. R. Herschbach, Angew. Chem., Int. Ed. Engl. 26, 1221 (1987).

[3] M. T. Bell and T. P. Softley, Mol. Phys. 107, 99 (2009).

[4] E. Narevicius and M. Raizen, Chem. Rev. 112, 4879 (2012).

[5] M. Brouard, D. H. Parker, and S. Y. T. van de Meerakker, Chem. Soc. Rev. 43, 7279 (2014).

[6] E. Roueff and F. Lique, Chem. Rev. 113, 8906 (2013).

[7] C. Naulin and M. Costes, Int. Rev. Phys. Chem. 33, 427 (2014).

[8] J. P. Toennies, W. Welz, and G. Wolf, J. Chem. Phys. 71, 614 (1979).

[9] D. W. Chandler, J. Chem. Phys. 132, 110901 (2010).

[10] N. Balakrishnan, A. Dalgarno, and R. C. Forrey, J. Chem. Phys. 113, 621 (2000).

[11] J. J. Gilijamse, S. Hoekstra, S. Y. T. van de Meerakker, G. C. Groeneboom, and G. Meijer, Science 313, 1617 (2006).

[12] M. Kirste, X. Wang, H. C. Schewe, G. Meijer, K. Liu, A. van der Avoird, L. M. C. Janssen, K. B. Gubbels, G. C. Groeneboom, and S. Y. T. van de Meerakker, Science 338, 1060 (2012).
[13] J. Onvlee, S. D. S. Gordon, S. N. Vogels, T. Auth, T. Karman, B. Nichols, A. van der Avoird, G. C. Groeneboom, M. Brouard, and S. Y. T. van de Meerakker, Nat. Chem. 9, 226 (2016).

[14] S. N. Vogels, J. Onvlee, S. Chefdeville, A. van der Avoird, G. C. Groeneboom, and S. Y. T. van de Meerakker, Science 350, 787 (2015).

[15] M. Costes and C. Naulin, Chem. Sci. 7, 2462 (2016).

[16] A. B. Henson, S. Gersten, Y. Shagam, J. Narevicius, and E. Narevicius, Science 338, 234 (2012).

[17] A. Osterwalder, EPJ Tech. Instrum. 2, 10 (2015).

[18] S. D. S. Gordon, J. Zou, S. Tanteri, J. Jankunas, and A. Osterwalder, Phys. Rev. Lett. 119, 053001 (2017).

[19] A. Klein, Y. Shagam, W. Skomorowski, P. S. Żuchowski, M. Pawlak, L. M. C. Janssen, N. Moiseyev, S. Y. T. van de Meerakker, A. van der Avoird, C. P. Koch, and E. Narevicius, Nat. Phys. 13, 35 (2017).

[20] P. Allmendinger, J. Deiglmayr, O. Schullian, K. Höveler, J. A. Agner, H. Schmutz, and F. Merkt, ChemPhysChem 17, 3596 (2016).

[21] S. Willitsch, M. T. Bell, A. D. Gingell, S. R. Procter, and T. P. Softley, Phys. Rev. Lett. 100, 043203 (2008).

[22] Y.-P. Chang, K. Długołecki, J. Küpper, D. Rösch, D. Wild, and S. Willitsch, Science 342, 98 (2013).

[23] B. C. Sawyer, B. K. Stuhl, M. Yeo, T. V. Tscherbul, M. T. Hummon, Y. Xia, J. Kłos, D. Patterson, J. M. Doyle, and J. Ye, Phys. Chem. Chem. Phys. 13, 19059 (2011).

[24] M Strebel, T.-O. Müller, B Ruff, F. Stienkemeier, and M. Mudrich, Phys. Rev. A 86, 062711 (2012).

[25] P. C. Zieger, S. Y. T. van de Meerakker, C. E. Heiner, H. L. Bethlem, A. J. A. van Roij, and G. Meijer, Phys. Rev. Lett. 105, 173001 (2010)

[26] P. C. Zieger, C. J. Eyles, G. Meijer, and H. L. Bethlem, Phys. Rev. A 87, 043425 (2013).

[27] P. C. Zieger, C. J. Eyles, S. Y. T. van de Meerakker, A. J. A. van Roij, H. L. Bethlem, and G. Meijer, Z. Phys. Chem. 227, 1605 (2013).

[28] U. Even, EPJ Tech. Instrum. 2, 17 (2015).

[29] L. Minnhagen, J. Opt. Soc. Am. 63, 1185 (1973).

[30] See Supplemental Material at http://link.aps.org/ supplemental/10.1103/PhysRevLett.120.033402 for an explanation how we correct for the fact that a small but significant fraction of the elastic collisions does not eject molecules from the ring.

[31] J. Loreau and A. van der Avoird, J. Chem. Phys. 143, 184303 (2015).

[32] C. Meng, A. P. P. van der Poel, C. Cheng, and H. L. Bethlem, Phys. Rev. A 92, 023404 (2015).

[33] N. R. Hutzler, H. Lu, and J. M. Doyle, Chem. Rev. 112, 4803 (2012).

[34] Y. Shagam and E. Narevicius, J. Phys. Chem. C 117, 22454 (2013).

[35] A. P. P. van der Poel, K. Dulitz, T. P. Softley, and H. L. Bethlem, New J. Phys. 17, 055012 (2015). 